\title{
CARDINAL ARITHMETIC IN THE STYLE OF BARON VON MÜNCHHAUSEN
}

\author{
ALBERT VISSER
}

\begin{abstract}
In this paper we show how to interpret Robinson's Arithmetic Q and the theory $\mathrm{R}$ of Tarski, Mostowski and Robinson as theories of cardinals in very weak theories of relations over a domain.
\end{abstract}

Bei der Verfolgung eines Hasen wollte ich mit meinem Pferd über einen Morast setzen. Mitten im Sprung musste ich erkennen, dass der Morast viel breiter war, als ich anfänglich eingeschätzt hatte. Schwebend in der Luft wendete ich daher wieder um, wo ich hergekommen war, um einen größeren Anlauf zu nehmen. Gleichwohl sprang ich zum zweiten Mal noch zu kurz und fiel nicht weit vom anderen Ufer bis an den Hals in den Morast. Hier hätte ich unfehlbar umkommen müssen, wenn nicht die Stärke meines Armes mich an meinem eigenen Haarzopf, samt dem Pferd, welches ich fest zwischen meine Knie schloss, wieder herausgezogen hätte. Baron von Münchhausen.

\section{INTRODUCTION}

The development of the arithmetic of the finite cardinals is one of the basic tasks of any foundational project. It is quite natural to ask ourselves with how little can we actually do $i t$ ? Of course, this question needs some further explication. We must ask ourselves what kind of means to develop arithmetic will we be considering? and how will we measure the strength of our solution?

We address the first question first. We propose to take the idea of cardinal seriously. A cardinal is given by an equivalence relation on classes. So, we need a theory of classes or relations as our starting point. We follow an idea of John Burgess (see [Bur05]): we start with a basic theory of binary relations over a domain of objects, adjunctive relation theory or ar. We consider what we should add to ar to get the weakest possible theories of numbers $\mathrm{R}$ and $\mathrm{Q}$. We will see that it is possible to derive both these theories from surprisingly modest additions to the basic theory ar.

As soon as one has $\mathrm{Q}$ one has automatically a lot more, e.g., the theory $I \Delta_{0}+\Omega_{1}$, as has been shown. a.o., by Edward Nelson. See his book [Nel86]. See also [HP91].

The theories of sets and relations that we will use as a basis to $\operatorname{develop} Q$ are all mutually interpretable. So, we need a more refined instrument to compare their strength. Fortunately, there is a well motivated method available. Our means of measuring strength is $\mathfrak{o}$-direct interpretability. This is an instrument appropriate to measure the strength of theories of such things as sets and classes over a given domain. We say that $V$ o-directly interprets $U$ if there is an interpretation $K$ of $U$ in $V$ that preserves both the object domain and the identity relation on the object domain. The idea is that we consider $U$ and $V$ as means to talk about the given domain. It is the job of our theories to treat the given domain. We only allow interpretations that preserve that basic functionality.

Date: February 6, 2009.

2000 Mathematics Subject Classification. 03A05, 03B30, 03F25,

Key words and phrases. interpretability, weak arithmetics, weak set theories. 
The interpretability of $\mathrm{Q}$ in a weak set theory has been studied extensively and we will make ample use of ideas and insights derived from previous results. Here is a brief history of the result that $Q$ is interpretable in the salient weak Adjunctive Set Theory or AS.

(1) In the paper [ST50], Wanda Szmielew and Alfred Tarski announce the interpretability of $\mathrm{Q}$ in a theory $\mathrm{S}$ that is essentially $\mathrm{AS}$ plus extensionality. ${ }^{1}$ See also [TMR53], p34. No proof was published.

(2) A proof of the Szmielew-Tarski result is given by George Collins and Joseph Halpern in [CH70]. Collins and Harper did not have Solovay's method of shortening cuts available. ${ }^{2}$ So, it is rather amazing that they manage to obtain a total addition and multiplication. They succeed by a clever choice of values for plus and times whenever the recursive definition does not turn out a value. Their interpretation of $Q$ is direct.

(3) Franco Montagna and Antonella Mancini, in their paper [MM94], give an improvement of the Szmielew-Tarski result. They prove that $Q$ can be interpreted in an extension $\mathrm{N}$ of $\mathrm{AS}$ in which we have the functionality of empty set and the operation of adjoining of singletons. They sketch a proof of the Herbrand consistency of their set theory that can be proved in a predicative arithmetic.

(4) In appendix III of [MPS90], Jan Mycielski, Pavel Pudlák and Alan Stern provide the ingredients of the interpretation of $\mathrm{Q}$ in $\mathrm{AS} .^{3}$ They do not develop the theory of addition and multiplication, but these can be treated in familiar ways using the theory of sequences that is provided by their argument. See e.g. [Pud83] or [HP91].

(5) John Burgess in his [Bur05], Section 2.2, provides a variant of the MontagnaMancini argument. Burgess starts with adjunctive relation theory ar (principles R1 and R2 on page 92 of Burgess' book), enriched with a theory of successor on the object domain (principles Q1 and Q2 on page 56 of Burgess' book).

In these proofs the basic operations are defined by recursion. We will provide a new recursion-free proof of the interpretability of $Q$ in AS.

The interpretability of $Q$ in AS important for more reasons than the foundational interest articulated above. It plays an important role in developing the notion of sequentiality, an explication of what it is for a theory to 'have coding'. See, e.g., the discussion of sequentiality in [Vis08].

\section{THEORIES AND INTERPRETATIONS}

In this section, we fix some basic concepts and notations. The reader is advised to go over it lightly, returning just when a notation or notion is not clear.

We work with RE theories in many-sorted first order predicate logic of finite signature. These theories have officially a relational signature. Unofficially, we use function symbols, but these can be eliminated using a well-known unwinding procedure. Every sort has identity.

The most general notion of interpretation is piecewise, more-dimensional, many-sorted, relative interpretations with parameters, where identity is not necessarily translated as identity. Since, the presence of parameters, being piecewise and more-dimensionality only play a minor role, we will only give a definition of one-dimensional, many-sorted, relative interpretations without parameters, where identity is not necessarily translated as identity.

\footnotetext{
${ }^{1}$ John Burgess in [Bur05], p90-91, calls this theory ST, for Szmielew-Tarski set theory.

${ }^{2}$ Solovay's method dates from roughly 1976. See the unpublished letter [Solle].

${ }^{3}$ Mycielski, Pudlák and Stern do not provide a name for their weak set theory. They call any theory that directly interprets AS: a weak set theory.
} 
2.1. Translations. To define an interpretation, we first need the notion of translation. Let $\Sigma$ and $\Xi$ be finite signatures for many-sorted predicate logic with finitely many sorts. The sorts are supposed to be specified with the signature. A relative translation $\tau: \Sigma \rightarrow \Xi$ is given by a triple $\langle\sigma, \delta, F\rangle$. Here $\sigma$ is a mapping of the $\Sigma$-sorts to the $\Xi$-sorts. The mapping $\delta$ assigns to every $\Sigma$-sort $\mathfrak{a}$ a $\Xi$-formula $\delta^{\mathfrak{a}}$ representing the domain for sort $\mathfrak{a}$ of the translation. We demand that $\delta^{\mathfrak{a}}$ contains at most a designated variable $v_{0}^{\sigma \mathfrak{a}}$ of sort $\sigma \mathfrak{a}$ free. The mapping $F$ associates to each relation symbol $R$ of $\Sigma$ a $\Xi$-formula $F(R)$. The relation symbol $R$ comes equipped a sequence $\overrightarrow{\mathfrak{a}}$ of sorts. We demand that $F(R)$ has at most the variables $v_{i}^{\sigma \mathfrak{a}_{i}}$ free. We translate $\Sigma$-formulas to $\Xi$-formulas as follows:

- $\left(R\left(y_{0}^{\mathfrak{a}_{0}}, \cdots, y_{n-1}^{\mathfrak{a}_{n-1}}\right)\right)^{\tau}:=F(R)\left(y_{0}^{\sigma \mathfrak{a}_{0}}, \cdots, y_{n-1}^{\sigma \mathfrak{a}_{n-1}}\right)$.

(We assume that some mechanism for $\alpha$-conversion is built into our definition of substitution to avoid variable-clashes.)

- $(\cdot)^{\tau}$ commutes with the propositional connectives;

- $\left(\forall y^{\mathfrak{a}} A\right)^{\tau}:=\forall y^{\sigma \mathfrak{a}}\left(\delta^{\mathfrak{a}}(y) \rightarrow A^{\tau}\right)$;

- $\left(\exists y^{\mathfrak{a}} A\right)^{\tau}:=\exists y^{\sigma \mathfrak{a}}\left(\delta^{\mathfrak{a}}(y) \wedge A^{\tau}\right)$.

Suppose $\tau$ is $\langle\sigma, \delta, F\rangle$. Here are some convenient conventions and notations.

- We write $\delta_{\tau}$ for $\delta$ and $F_{\tau}$ for $F$.

- We write $R_{\tau}$ for $F_{\tau}(R)$.

- We will always use $"={ }^{\mathfrak{a}}$ for the identity relation of a theory for sort $\mathfrak{a}$. In the context of translating, we will however switch to ' $E^{\mathfrak{a}}$ ',

- We write $\vec{x}: \delta^{\overrightarrow{\mathfrak{a}}}$ for: $\delta^{\mathfrak{a}_{0}}\left(x_{0}^{\sigma \mathfrak{a}_{0}}\right) \wedge \ldots \wedge \delta^{\mathfrak{a}_{n-1}}\left(x_{n-1}^{\sigma \mathfrak{a}_{n-1}}\right)$.

- We write $\forall \vec{x}: \delta^{\overrightarrow{\mathfrak{a}}} A$ for: $\forall x_{0}^{\sigma \mathfrak{a}_{0}} \ldots \forall x_{n-1}^{\sigma \mathfrak{a}_{n-1}}\left(\vec{x}: \delta^{\overrightarrow{\mathfrak{a}}} \rightarrow A\right)$. Similarly for the existential case.

2.2. Interpretations and Interpretability. A translation $\tau$ supports a relative interpretation of a theory $U$ in a theory $V$, if, for all axioms $A$ of $U$, we have $U \vdash A \Rightarrow V \vdash A^{\tau}$. (Note that this automatically takes care of the theory of identity. Moreover, it follows that $V \vdash \exists v_{0} \delta_{\tau}^{\mathfrak{a}} v_{0}$, for each $\Sigma$-sort a.) Thus, an interpretation has the form: $K=\langle U, \tau, V\rangle$.

Par abus de langage, we write ' $\delta_{K}$ ' for: $\delta_{\tau_{K}}$; ' $P_{K}$ ' for: $P_{\tau_{K}}$; ' $A^{K}$ ' for: $A^{\tau_{K}}$, etc. We define:

- We write $K: U \triangleleft V$ or $K: V \triangleright U$, for: $K$ is an interpretation of the form $\langle U, \tau, V\rangle$.

- $V \triangleright U: \Leftrightarrow U \triangleleft V: \Leftrightarrow \exists K K: U \triangleleft V$. $U$.

We read $U \triangleleft V$ as: $U$ is interpretable in $V$. We read $V \triangleright U$ as: $V$ interprets

We say that a theory $V$ locally interprets a theory $U$ if, for any finite subtheory $U_{0}$ of $U$, we have $V \triangleright U_{0}$. We write $V \triangleright_{\text {loc }} U$ for: $V$ locally interprets $U$.

2.3. Special Interpretations. We consider pointed theories, i.e., theories with a designated sort of objects $\mathfrak{o}$. An translation $\tau$ is $\mathfrak{o}$-direct if $\sigma_{\tau}$ preserves the designated sort, and, for the designated sort, $\tau$ is unrelativized and has absolute identity, i.e.:

- $\delta^{\mathfrak{o}}\left(v_{0}\right): \leftrightarrow\left(v_{0}=v_{0}\right)$,

- $v_{0} E_{\tau}^{\mathfrak{o}} v_{1}: \leftrightarrow v_{0}=v_{1}$.

An interpretation is $\mathfrak{o}$-direct if it is based on a direct translation. We write $V \triangleright_{\mathfrak{o}}$-dir $U$, for $V$ o-directly interprets $U$, etc.

We will use the o-direct sum $T \boxplus_{\mathfrak{o}} U$ of pointed theories. This sum is obtained as follows. First we make the sorts and predicates of the theories disjoint except the designated sort $\mathfrak{o}$ and except for the identity predicates for the designated sort. Then, we take the union of the modified theories.

Our sum is the sum in a suitable category of $\mathfrak{o}$-direct interpretations. Thus, the sum is a bifunctor w.r.t. the preorder of direct interpretability, i.e., if $V \triangleright_{\mathfrak{o} \text {-dir }} U$ and $V^{\prime} \triangleright_{\mathfrak{o} \text {-dir }} U^{\prime}$, then $\left(V \boxplus_{\mathfrak{o}} V^{\prime}\right) \triangleright_{\mathfrak{o} \text {-dir }}\left(U \boxplus_{\mathfrak{o}} U^{\prime}\right)$. 
The one-sorted theory of pure equality $E Q$ is in the lowest degree of $\mathfrak{o}$-direct interpretability together with any many-sorted predicate logic. We clearly have $\mathrm{EQ} \boxplus_{\mathfrak{o}} U=U$.

In case we consider one-sorted theories, we will use the default assumption that the sort is $\mathfrak{o}$.

\section{TheOries of Number}

In this section we discuss various weak theories of number and their interrelations. First, we formulate systems with partial operations that can also be theories of a number, and, then, we give the traditional systems $R$ and $Q$ with total operations. The systems for partial operations will emerge as the natural systems for cardinal arithmetic in our context. In the rest of the paper, we will work with these systems. In this section, we will pin down the precise relationships of certain systems of partial operations and $R$ and $Q$.

3.1. Theories of a Number of all Numbers. We formulate theories that can be a both theories of a number and of all numbers.

The theory $\mathrm{TN}_{0}$ is given a follows. We have, apart from equality, the following symbols in the signature: a constant 0 , one binary relation symbols $\mathrm{S}$, and two ternary relations $\mathrm{A}$ and $\mathrm{M}$. The theory is axiomatized as follows.

$$
\begin{aligned}
\operatorname{tn} 1 & \vdash(\mathrm{S} x y \wedge \mathrm{S} u v) \rightarrow((x=u \wedge y=v) \vee(x \neq u \wedge y \neq v)), \\
\operatorname{tn} 2 & \vdash \neg \mathrm{S} x 0, \\
\operatorname{tn} 3 & \vdash x=0 \vee \exists y \mathrm{~S} y x \\
\operatorname{tn} 4 & \vdash(\mathrm{A} x y u \wedge \mathrm{A} x y v) \rightarrow u=v, \\
\operatorname{tn} 5 & \vdash \mathrm{A} x 0 x, \\
\operatorname{tn} 6 & \vdash \exists u(\mathrm{~S} y u \wedge \mathrm{A} x u v) \leftrightarrow \exists z(\mathrm{~S} z v \wedge \mathrm{A} x y z), \\
\operatorname{tn} 7 & \vdash(\mathrm{M} x y u \wedge \mathrm{M} x y v) \rightarrow u=v, \\
\operatorname{tn} 8 & \vdash \mathrm{M} x 00, \\
\operatorname{tn} 9 & \vdash x \neq 0 \rightarrow(\exists u(\mathrm{~S} y u \wedge \mathrm{M} x u v) \leftrightarrow \exists z(\mathrm{~A} z x v \wedge \mathrm{M} x y z)), \\
\text { We will treat } \leqslant \text { as a defined relation: } & \\
& \bullet \vdash x \leqslant y: \leftrightarrow \exists z \mathrm{~A} z x y .
\end{aligned}
$$

We briefly pause to see the necessity of the assumption of Axiom tn9. Suppose we would drop it. We get, from the resulting axiom in combination with Axiom tn 5 , taking $x, v, z:=$ 0 :

$$
\vdash \mathrm{M} 0 y 0 \rightarrow \exists u(\mathrm{~S} y u \wedge \mathrm{M} 0 u 0) .
$$

It follows that the class of $y$ such that $M 0 y 0$ contains 0 and is closed under successor. Hence, we can derive $\mathrm{E}_{n}^{\text {num }}:=\exists x_{1}, \ldots, x_{n}\left(S 0 x_{1} \wedge \ldots \wedge S x_{n-1} x_{n}\right)$, for each $n{ }^{4}$ This defeats our intention to give a theory that can also be the theory of a number.

We define the theory $\mathrm{TN}_{n}$ as $\mathrm{TN}_{0}$ plus the axiom $\mathrm{E}_{n}^{\text {num }}$. We define $\mathrm{TN}_{\infty}$ as $\mathrm{TN}_{0}$ pus the axioms $\mathrm{E}_{n}^{\text {num }}$, for every $n$.

The following theorem, is not really necessary for the subsequent development, but it gives some feeling for the theory $\mathrm{TN}_{0}$. Let INF be the theory axiomatized by the axioms $\mathrm{E}_{n}^{\mathrm{ob}}:=\exists x_{0}, \ldots, x_{n-1} \bigwedge_{i<j} x_{i} \neq x_{j}$.

Theorem 3.1. The theories $\mathrm{TN}_{n}$ and $\mathrm{TN}_{0}+\mathrm{E}_{n}^{\mathrm{ob}}$ coincide. It follows that $\mathrm{TN}_{\infty}$ and $\mathrm{TN}_{0}+$ INF coincide.

Proof. It is easy to see that $\mathrm{E}_{n}^{\text {num }}$ implies $\mathrm{E}_{n}^{\text {ob }}$ over $\mathrm{TN}_{0}$.

We show that $\mathrm{TN}_{0}$ plus $\mathrm{E}_{n}^{\text {ob }}$ proves $\mathrm{E}_{n}^{\text {num }}$. Reason in $\mathrm{TN}_{0}$. Suppose we have $\mathrm{S} v_{0} v_{1}$, $\ldots, \mathrm{S} v_{k-2} v_{k-1}$. We claim that $\mathrm{E}_{k}^{\text {num }}$, i.e., there are $u_{1}, \ldots, u_{k-1}$ such that $\mathrm{S} 0 u_{1}, \ldots$, $\mathrm{S} u_{k-2} u_{k-1}$. This follows from Axiom tn5 plus repeated applications of Axiom tn6. Clearly,

\footnotetext{
${ }^{4}$ We can, in fact, do much more. By Solovay's methods, we can interpret $\mathrm{AS}_{0}$ plus the axiom that $\mathrm{S}$ is total. This theory interprets Q. See Subsection 3.3.
} 
we have $\mathrm{S} v_{0} v_{1}$ and $\mathrm{A} v_{0} 0 v_{0}$. Hence, for some $u_{1}, \mathrm{~S} 0 u_{1}$ and $\mathrm{A} v_{0} u_{1} v_{1}$. Now we have $\mathrm{S} v_{1} v_{2}$ and $\mathrm{A} v_{0} u_{1} v_{1}$. Hence, for some $u_{2}, \mathrm{~S} u_{1} u_{2}$ and $\mathrm{A} v_{0} u_{2} v_{2}$. Etc.

Suppose we have pairwise distinct elements $x_{0}, \ldots, x_{n-1}$. Consider $x_{i}$. By Axiom tn3, $x_{i}$ is either 0 or a successor. In the second case it has a predecessor $x_{i}^{(1)}$. Similarly, $x_{i}^{(1)}$ is either 0 or it has a predecessor $x_{i}^{(2)}$. Etc. If we have a descending chain of length $n$, it follows by our previous observation that $\mathrm{E}_{n}^{\text {num }}$. If, for all $i$, the descending chain is smaller than $n$, then it follows by the functionality and injectivity of successor that the $x_{i}$ are among the elements of some chain $\mathrm{S} w_{0} w_{1}, \ldots, \mathrm{S} w_{j-2} w_{j-1}$, for some $j<n$. Quod impossibile, since the $x_{j}$ are pairwise disjoint.

3.2. The theory R. The theory $\mathrm{R}$ was introduced by Tarski, Mostowski and Robinson in their book [TMR53]. It is a very weak theory that is essentially undecidable, i.e., every consistent RE extension of the theory is undecidable. It was observed by Cobham that one still has an essentially undecidable theory if one drops the axiom R6 (given below), obtaining the theory $\mathrm{R}_{0}$. See [Vau62] and [JS83]. Cobham has shown that $\mathrm{R}$ has a stronger property. Consider any RE theory $T$. Suppose we have translation $\alpha$ of the arithmical language into the language of $T$. Suppose $T$ is consistent with $\mathrm{R}_{0}^{\alpha}$. Then, $T$ is undecidable. ${ }^{5}$ For the proof of a closely related result, see Vaught's paper [Vau62]. In fact one can show that, if $T$ is consistent with $\mathrm{R}_{0}^{\alpha}$, then there is a finitely axiomatized extension $A$ of $\mathrm{R}_{0}$ and a translation $\beta$, such that $T$ is consistent with $A^{\beta}$.

We consider the signature with $0, \mathrm{~S},+$ and $\cdot$ We define $\underline{0}:=0, \underline{n+1}:=\mathrm{S} \underline{n}$, and $x \leqslant y: \leftrightarrow \exists z z+x=y$. We have the following axioms.

R1. $\vdash \mathrm{S} \underline{n}=\underline{n+1}$

R2. $\vdash \underline{m}+\underline{n}=\underline{m}+n$

R3. $\vdash \underline{m} \cdot \underline{n}=\underline{m \cdot n}$

R4. $\vdash \underline{m} \neq \underline{n}, \overline{\text { for } m} \neq n$

R5. $\vdash x \leqslant \underline{n} \rightarrow \bigvee_{i \leqslant n} x=\underline{i}$

R6. $\vdash x \leqslant \underline{n} \vee \underline{n} \leqslant x$

The theory $\mathrm{R}_{0}$ is axiomatized by $\mathrm{R} 1-5$ and $\mathrm{R}$ is axiomatized by $\mathrm{R} 1-6 .{ }^{6}$

We first show that $\mathrm{TN}_{\infty}$ interprets $\mathrm{R}$. We define a translation $\sigma$.

- $\delta_{\sigma} v: \leftrightarrow v=v$

- $\mathrm{S}_{\sigma} v w: \leftrightarrow \mathrm{S} v w \vee(\forall u \neg \mathrm{S} v u \wedge w=0)$

- $\mathrm{A}_{\sigma} v_{0} v_{1} w: \leftrightarrow \mathrm{A} v_{0} v_{1} w \vee\left(\forall u \neg \mathrm{A} v_{0} v_{1} u \wedge w=0\right)$,

- $\mathrm{M}_{\sigma} v_{0} v_{1} w: \leftrightarrow \mathrm{M} v_{0} v_{1} w \vee\left(\forall u \neg \mathrm{M} v_{0} v_{1} u \wedge w=0\right)$

It is easily seen that $\sigma$ gives us an interpretation of $\mathrm{R}$ in $\mathrm{TN}_{\infty}$.

We proceed to provide an interpretation of $T N_{\infty}$ in $R_{0}$. Since, everything is simple once we have a decent linear ordering on our objects, we will first develop an extension of $R_{0}$ with a linear ordering. Our development is an extension of the one given in [JS83].

We will employ the notational machinery of virtual classes. We define $[x, y]:=\{z \mid x \leqslant$ $z \leqslant y\}$ and $x<y: \leftrightarrow x \leqslant y \wedge x \neq y$. We define the virtual class $X$ as follows. It is the class of those $x$ such that:

$\mathrm{X} 1 .[0, x]$ is a linear ordering that contains 0 and $x$

$\mathrm{X} 2 . u<v \leqslant x \rightarrow \mathrm{S} u \leqslant v$;

X3. $u \leqslant v \leqslant x \rightarrow \exists w \leqslant v w+u=v$;

Lemma $3.1\left(\mathrm{R}_{0}\right)$. We have:

\footnotetext{
${ }^{5}$ Cobham's proof remains unpublished, but, using the methods and results of this paper and the clues provided in [Vau62], it is not hard to find a proof.

${ }^{6}$ The original version of $\mathrm{R}$ does not have $\mathrm{S}$, but a constant 1 . However it is definitionally equivalent with our version: The original version can be recovered from ours by translating 1 to S0. Our version can be recovered from the original one by translating $\mathrm{S} x$ to $x+1$.
} 
i. $\underline{n} \in X$.

ii. $X$ is downwards closed w.r.t. $\leqslant$.

iii. For $x, y \in X$, we have $x \leqslant y \leftrightarrow \exists z \in X z+x=y$.

Proof. (i) and (ii) are trivial. We treat (iii). From right to left is trivial. Suppose $x, y \in X$ and $x \leqslant y$. since $y \in[0, y]$ and $[0, y]$ is a linear ordering, we have $x \leqslant y \leqslant y$, and, hence, there is a $z \leqslant y$, such that $z+x=y$. Since $X$ is downwards closed by (ii), we find $z \in X$.

We now construct a translation $\tau$ by:

- $\delta_{\tau}:=X$,

- $0_{\tau}:=0$,

- $\mathrm{S}_{\tau} x:=\mathrm{S} x$, if $\mathrm{S} x \in X, \mathrm{~S}_{\tau} x:=x$, otherwise.

- $x+{ }_{\tau} y:=x+y$, if $x+y \in X, x+{ }_{\tau} y:=y$, otherwise,

- $x \cdot{ }_{\tau} y:=x \cdot y$, if $x \cdot y \in X, x \cdot{ }_{\tau} y:=y$, otherwise,

The translation $\tau$ gives us an interpretation of $\mathrm{R}_{0}$ plus the following axioms.

- $\leqslant$ is a treelike partial ordering, i.e., it is a partial ordering satisfying $y \leqslant x$ and $z \leqslant x$ implies $y \leqslant z$ or $z \leqslant y$

- $\vdash y<x \rightarrow \mathrm{S} y \leqslant x$

- $\vdash y \leqslant x \rightarrow \exists z \leqslant x z+x=y$

The only worry in the verification is the absoluteness of $\leqslant$ w.r.t. $\tau$. Consider $x, y \in X$. If $x \leqslant y$, then there is a $z \in X$, such that $z+x=y$, and hence $z+{ }_{\tau} x=y$. So, $x \leqslant{ }_{\tau} y$. Conversely, suppose $x \leqslant_{\tau} y$. So, for some $z \in X, z+_{\tau} x=y$. In case $z+x \in X$, we have $z+x=y$, so $x \leqslant y$ and we are done. If $z+x \notin X$, we have $x=y$. Since $y \in X$, we have $y \leqslant y$, and so $x \leqslant y$.

Let's call the theory so obtained $\mathrm{R}_{1}$. We work in $\mathrm{R}_{1}$. Define $Y$ as the class of all $x$, such that $(Y 1)$ : for all $y, y \leqslant x$ or $x \leqslant y$.

Lemma $3.2\left(\mathrm{R}_{1}\right)$. We have:

i. $\underline{n} \in Y$.

ii. $\bar{Y}$ is downwards closed.

iii. $\leqslant$ restricted to $Y$ is a linear ordering.

Proof. We prove (i). Consider any $y$. We prove $y \leqslant \underline{n}$ or $\underline{n} \leqslant y$, by external induction on $n$. Since $[0, y]$ is a linear ordering including 0 and $y$, we have $0 \leqslant y$. Suppose we have $y \leqslant \underline{n}$ or $\underline{n} \leqslant y$. In the first case, $y \leqslant \underline{n} \leqslant n+1$, and hence $y \leqslant n+1$. In the second case, we have either $\underline{n}=y$ or $\underline{n} \neq y$. In the first subcase, we find $y=\underline{n} \leqslant \underline{n}+1$. In the second subcase, we find $\underline{n}<y$, and, hence, $\underline{n+1}=\mathrm{S} \underline{n} \leqslant y$.

We prove (ii). Suppose $z \leqslant x \in Y$. Consider any $y$. We have $y \leqslant x$ or $x \leqslant y$. In the first case, $y \leqslant z$ or $z \leqslant y$, since $[0, x]$ is linear. In the second case, $z \leqslant x \leqslant y$, so $z \leqslant y$.

(iii) is trivial.

We define a translation $\rho$ as follows.

- $\delta_{\rho}:=Y$,

- $0_{\rho}:=0$,

- $\mathrm{S}_{\rho} x:=\mathrm{S} x$, if $\mathrm{S} x \in Y, \mathrm{~S}_{\rho} x:=x$, otherwise.

- $x+{ }_{\rho} y:=x+y$, if $x+y \in Y, x+{ }_{\rho} y:=y$, otherwise,

- $x \cdot{ }_{\rho} y:=x \cdot y$, if $x \cdot y \in Y, x \cdot{ }_{\rho} y:=y$, otherwise,

It is easy to see that the interpretation based on $\rho$ gives us the theory $\mathrm{R}^{\star}$, axiomatized by:

$\mathrm{R}^{\star} 1 . \vdash \mathrm{S} \underline{n}=\underline{n+1}$

$\mathrm{R}^{\star} 2 . \vdash \underline{m}+\underline{n}=\underline{m}+n$

$\mathrm{R}^{\star} 3 . \vdash \underline{m} \cdot \underline{n}=\underline{m \cdot n}$ 
$\mathrm{R}^{\star} 4 . \vdash \underline{m} \neq \underline{n}$, for $m \neq n$

$\mathrm{R}^{\star} 5 . \vdash \bar{x} \leqslant \underline{n} \rightarrow \bigvee_{i \leqslant n} x=\underline{i}$

$\mathrm{R}^{\star} 6 . \leqslant$ is a linear ordering

$\mathrm{R}^{\star} 7 . \vdash x<y \rightarrow \mathrm{S} x \leqslant y$

$\mathrm{R}^{\star} 8 . \vdash x \leqslant y \rightarrow \exists z \leqslant y z+x=y$

Finally, we define the promised interpretation of $\mathrm{TN}_{\infty}$. Let $W$ be any virtual class (possibly with parameters). We define a translation $\theta_{W}$ of the language of $\mathrm{TN}_{0}$, as follows.

- $\delta_{\theta, W}:=W$,

- $0_{\theta, W}:=0$,

- $\mathrm{S}_{\theta, W} v w: \leftrightarrow v, w \in W \wedge \mathrm{S} v=w$,

- $\mathrm{A}_{\theta, W} v_{0} v_{1} w: \leftrightarrow v_{0}, v_{1}, w \in W \wedge v_{0}+v_{1}=w$,

- $\mathrm{M}_{\theta, W} v_{0} v_{1} w: \leftrightarrow v_{0}, v_{1}, w \in W \wedge v_{0} \cdot v_{1}=w$,

We write $W \models B$ for $B^{\theta_{W}}$.

We work in $\mathrm{R}^{\star}$. Define $Z:=\left\{x \mid \forall y \leqslant x[0, y] \models \mathrm{TN}_{0}\right\}$. It is easy to see that each $\underline{n}$ is in $Z$.

We take $\theta_{Z}$ as our desired interpretation of $\mathrm{TN}_{\infty}$. Clearly, for each $n, Z \models \mathrm{E}_{n}^{\text {num }}$. Moreover $Z$ is downwards closed.

We check one direction of one sample axiom of $\mathrm{TN}_{0}$, to wit $\operatorname{tn} 6$. The other verifications are similar. Suppose, we have $x, y, u, v \in Z$ and $\mathrm{S} y=u$ and $x+u=v$. Let $w$ be the maximum of $x, y, u, v$. Since $[0, w] \models \mathrm{TN}_{0}$, we find $\exists z \leq w(\mathrm{~S} z=v \wedge x+y=z)$. By the downwards closure of $Z$, we find that $z \in Z$.

3.3. The Theory Q. Robinson's Arithmetic Q was introduced in [TMR53]. It is a finitely axiomatized sequential theory. Using Solovay's method of shortening cuts (see [Solle]), one can show that $Q$ interprets seemingly much stronger theories like $I \Delta_{0}+\Omega_{1}$. See [Nel86] and [HP91]. Here are the axioms of $\mathbf{Q}$.

Q1. $\vdash \mathrm{S} x=\mathrm{S} y \rightarrow x=y$,

Q2. $\vdash 0 \neq \mathrm{S} x$,

Q3. $\vdash x=0 \vee \exists y x=\mathrm{S} y$,

Q4. $\vdash x+0=x$,

Q5. $\vdash x+\mathrm{S} y=\mathrm{S}(x+y)$,

Q6. $\vdash x \times 0=0$,

Q7. $\vdash x \times \mathrm{S} y=x \times y+x$.

The theory $\mathrm{Q}$ is mutually interpretable with $T \mathrm{~N}_{0}$ plus the axiom that $\mathrm{S}$ is total, by a result of Vítěslav Švejdar. The theory $T N_{0}$ plus $S$ is total is Hájek's weak arithmetic $Q_{\text {haj }}^{-}$. The theory $Q_{\text {haj }}^{-}$extends an even weaker theory $Q^{-}$, due to Grzegorczyk. Švejdar, in his paper [Šve07], shows that $Q$ is interpretable in $Q^{-}$.

\section{Adjunctive Relation Theory}

We define adjunctive relation theory, ar, as follows. The theory ar is two-sorted, with a sort $\mathfrak{o}$ of objects and a sort $\mathfrak{r}$ of binary relations. We have a ternary application predicate app of type roo. We write ' $R x y$ ' or ' $(x, y) \in R$ ' for: $\operatorname{app}(R, x, y){ }^{7}$

ar1. $\vdash \exists R \forall x, y \neg R x y$,

ar2. $\vdash \forall R, x, y \exists S \forall u, v(S u v \leftrightarrow(R u v \vee(u=x \wedge v=y))$.

ar3. $\vdash R=S \leftrightarrow \forall x, y(R x y \leftrightarrow S x y)$.

\footnotetext{
${ }^{7}$ We use minuscules in the name 'ar' to stress the non-iterability of our relations. This theory allows finite models. We will write e.g. the name of adjunctive set theory 'AS' in majuscules, since the sets we consider are iterable. This theory only has infinite models.
} 
The theory ar was introduced by John Burgess in his book [Bur05]. Note that extensional identity on relations can be added as a defined relation to ar. It is then a theorem that extensional identity functions as a congruence w.r.t. app. Note, however that by having ar3, we do put a constraint on extensions of the theory in an expanded signature.

To formulate comprehension principles. it will be useful to treat the theory in a richer signature. If the additional signature is $\Sigma$, we will call the theory $\operatorname{ar}_{\Sigma}$.

We will use the usual set theoretic abbreviations on relations, like $\emptyset$ for the empty relation, $R \cup\{(x, y)\}^{8}$, for adjunction, $\subseteq, \cup$, etc.

We will develop $\mathfrak{o}$-direct interpretations of stronger versions of the theory. The methodology of our bootstrap is as follows. First, we will follow the cardinal style of development. Secondly, we aim at the very weak arithmetics $R$ and $Q_{\text {haj }}^{-}$. The strategy has the advantage that we obtain a good modularity of the development. The basic results work independent of whether the domain is finite or infinite. The choice to aim at $\mathrm{Q}_{\text {haj }}^{-}$has one disadvantage: it obscures that, in the theory ar + nu (to be introduced later), we can develop a category of classes over the basic objects with all finite limits, initial objects and finite sums. (We did not try to build co-equalizers, so it is not clear whether we can get all finite co-limits too.)

4.1. Downwards Closure of Virtual Classes. We first introduce a useful operation on virtual classes and show that this operation preserves desirable properties.

We work in $\operatorname{ar}_{\Sigma}$. Let $\mathcal{Y}$ be any virtual class of relations that (i) contains the empty relation and (ii) is closed under adjunction and (iii) is such that, for all $R \in \mathcal{Y}$, and all $S$, $R \cap S$ exists. Define $\operatorname{sub}(\mathcal{Y})$ as the class of all $R$ such that, for all $S \subseteq R$ we have $S \in \mathcal{Y}$.

We show that $\operatorname{sub}(\mathcal{Y})$ contains the empty relation, is closed under adjunction, and is downwards closed under $\subseteq$.

We treat the case of closure under adjunction. Suppose $R$ is in $\operatorname{sub}(\mathcal{Y})$ and $S \subseteq$ $R \cup\{(x, y)\}$. Clearly, $R \cap S$ exists and $(R \cap S) \subseteq R$. We may conclude that $R \cap S$ is in $\mathcal{Y}$. Either $S=R \cap S$ or $S=(R \cap S) \cup\{(x, y)\}$. In both cases, we find that $S$ is in $\mathcal{Y}$.

4.2. Bounded Predicative Comprehension. A formula of $\operatorname{ar}_{\Sigma}$ is predicative if it contains no bound class variables.

We work in $\operatorname{ar}_{\Sigma}$. Let $A_{i}(x, y, \vec{z}, \vec{Q})$ be a finite sequence of formulas. We demand that $A_{0}(x, y, Q)=Q x y$, for some chosen variable $Q$. Consider the virtual class $\mathcal{P}_{0}$ of all binary relations $P$ such that $\bigwedge_{i} \forall \vec{z}, \vec{Q} \exists R \forall x, y\left(R x y \leftrightarrow\left(P x y \wedge A_{i}(x, y, \vec{z}, \vec{Q})\right)\right)$. It is easy to see that $\mathcal{P}_{0}$ is contains the empty relation and is closed under adjunction.

Consider any $P$ in $\mathcal{P}_{0}$, and any $Q$. By the definition of $\mathcal{P}_{0}$ and the special choice of $A_{0}$, we can find an $R$ such that, for all $x, y, R x y$ iff $P x y$ and $Q x y$. I.o.w., $P \cap Q$ exists.

We take $\mathcal{P}:=\operatorname{sub}\left(\mathcal{P}_{0}\right)$. When we relativize our relations to $\mathcal{P}$, we $\mathfrak{o}$-directly interpret $\operatorname{ar}_{\Sigma}$ plus Bounded Predicative Comprehension $\mathrm{BPC}_{\Sigma}$ for the formulas $A_{i}$ :

$\mathrm{BPC}_{A_{i}} . \vdash \forall \vec{z}, P, \vec{Q} \exists R \forall x, y\left(R x y \leftrightarrow\left(P x y \wedge A_{i}(x, y \vec{z}, \vec{Q})\right)\right)$.

Note that we need downwards closure to guarantee that the promised relation $R$ is indeed in $\mathcal{P}$.

We may conclude that $\operatorname{ar}_{\Sigma} \triangleright_{\mathfrak{o}-\text { dir,loc }}\left(\operatorname{ar}_{\Sigma}+\mathrm{BPC}_{\Sigma}\right)$.

Inspection of our argument shows that we can even do a bit better: we can allow subsetbounded quantifiers in the formulas of our comprehension principle.

In this paper we will only use a finitely many instances of BPC. Still it is pleasant not to have to worry about which precise instances one needs. We will reason with the full principle in the background. When a result is reached, we will note that we used only finitely many instances, so that we proved global and not merely local interpretability.

\footnotetext{
${ }^{8}$ We use ' $(x, y)$ ' for external, non-iterable pairing and we use ' $\langle x, y\rangle$ ' for the internal, iterable pairing that we have in some theories.
} 
4.3. Union. We work in $\operatorname{ar}_{\Sigma}+\mathrm{BPC}_{\Sigma}$. Consider the class $\mathcal{U}$ of $R$ such that, for all $S$, we have $R \cup S$ exists. Clearly, $\mathcal{U}$ contains the empty relation and all singleton relations.

We show that $\mathcal{U}$ is closed under unions. Suppose $X$ and $Y$ are in $\mathcal{U}$. Clearly, $Y \cup Z$ exists and, hence, $X \cup(Y \cup Z)$ exists. Since $(X \cup Y) \cup Z=(X \cup(Y \cup Z))$, we find that $(X \cup Y) \cup Z$ exists.

Finally, we show that $\mathcal{U}$ is downwards closed w.r.t. $\subseteq$. Suppose $Y \subseteq X \in \mathcal{U}$. Then $Y \cup Z=\{w \in(X \cup Z) \mid w \in Y \vee w \in Z\}$ exists by $\mathrm{BPC}_{\Sigma}$.

By relativizing our relations to $\mathcal{U}$, we get an $\mathfrak{o}$-direct interpretation $\operatorname{of}_{\Sigma} \operatorname{ar}_{\Sigma}^{+}:=\operatorname{ar}_{\Sigma}+$ $\mathrm{BPC}_{\Sigma}+$ union, where union is the axiom that unions exist.

4.4. Classes and Functions. We define classes as diagonal relations, writing $x \in X$ for $X x x$. We adopt the usual abbreviations like $\subseteq$ and $\cap$ for classes. We write, using ' $f$ ' to range over relations:

$$
f: X \rightarrow Y \text { for: } \forall x(x \in X \leftrightarrow \exists y f x y) \wedge \forall x, y, y^{\prime}\left(\left(f x y \wedge f x y^{\prime}\right) \rightarrow y=y^{\prime}\right) .
$$

If $f: X \rightarrow Y$, we often write $f x=y$, for $f x y$.

Note that, if $X \subseteq Y$, then there is a function $\operatorname{emb}_{X, Y}: X \rightarrow Y$, witnessing the embedding: this is just $X$ itself as a diagonal function. Moreover, in $\operatorname{ar}_{\Sigma}^{+}$, we have that, for $f: X \rightarrow Y, X^{\prime} \subseteq X, Y^{\prime} \subseteq Y$ :

(1) $f\left[X^{\prime}\right]$, the $f$-image of $X^{\prime}$ exists.

(2) $f^{-1}\left[Y^{\prime}\right]$, the inverse $f$-image of $Y^{\prime}$ exists.

(3) $f \uparrow X^{\prime}: X^{\prime} \rightarrow Y$ exists.

(4) $f 1 Y^{\prime}: f^{-1}\left[Y^{\prime}\right] \rightarrow Y^{\prime}$ exists.

4.5. Creating a Category. We work in $\operatorname{ar}_{\Sigma}^{+}$. Define the virtual class $\mathcal{X}_{0}$ as the class of all classes $X$ such that:

i. for all $f: X \rightarrow Y, g: Y \rightarrow Z$, there is a $h: X \rightarrow Z$ such that, for all $x \in X$, $h x=g f x$, i.o.w. $g \circ f$ exists;

ii. for all $y$, the function $\mathrm{c}_{X, y}: X \rightarrow\{y\}$ with $\mathrm{c}_{X, y} x:=y$, exists;

iii. if $f: X \rightarrow Y$ is a bijection then $f$ is an isomorphism, i.e., the inverse $f^{-1}$ exists.

Clearly, the empty class and al singletons are in $\mathcal{X}_{0}$. Suppose $X$ and $Y$ are in $\mathcal{X}_{0}$. We show that $X \cup Y$ is in $\mathcal{X}_{0}$.

We treat case (i). Suppose $f:(X \cup Y) \rightarrow Z$ and $g: Z \rightarrow W$. We now may take: $g \circ f=(g \circ(f \uparrow X)) \cup(g \circ(f \uparrow Y))$.

Case (ii) is easy. We treat Case (iii). Suppose we have a bijection $f$ between $X \cup Y$ and $Z$. It follows that $f_{0}:=(f \uparrow X) 1 f[X]$ is a bijection between $X$ and $f[X]$. Also, $f_{1}:=(f \uparrow Y) 1 f[Y]$ is a bijection between $Y$ and $f[Y]$. Let $g_{0}$ and $g_{1}$ be the promised inverses of $f_{0}$, respectively $f_{1}$. Then $\left(g_{0} \cup g_{1}\right): Z \rightarrow(X \cup Y)$ is an inverse of $f$.

Let $\mathcal{X}:=\operatorname{sub}\left(\mathcal{X}_{0}\right)$. Clearly $\mathcal{X}$ contains the empty set and all singletons. We show that $\mathcal{X}$ is closed under unions. Suppose $X$ and $Y$ are in $\mathcal{X}$ and $Z \subseteq(X \cup Y)$. It follows that $(Z \cap X) \subseteq X$ and $(Z \cap Y) \subseteq Y$. Ergo, $(Z \cap X) \in \mathcal{X}_{0}$ and $(Z \cap Y) \in \mathcal{X}_{0}$. it follows that $Z=((Z \cap X) \cup(Z \cap Y)) \in \mathcal{X}_{0}$.

We define a category with as objects the elements of $\mathcal{X}$ and as morphisms the functions $f: X \rightarrow Y$. (Strictly speaking, the morphisms are the triples $(X, f, Y)$.) It is easy to verify that this is a category with as initial object the empty set and with as final objects the singletons. Also, it is easy to see that the category has equalizers.

We redefine the notion of class to: class in $\mathcal{X}$. So, from this point on, ' $X$ ', ' $Y$ ', ' $Z$ ' will range over $\mathcal{X}$.

In the following two subsections we verify the desired properties of the sum and the product. This just follows the standard development. The main point of the verification is to convince ourselves that $\operatorname{ar}_{\Sigma}^{+}$is sufficient to make all the steps work. 
4.6. The Sum. Remarkably, the treatment we give below only works when we have at least two elements in the object domain. If there is only one object, we do not get the injectivity of successor, since we will have $\mathrm{S} 0=\mathrm{S} 1=1$. For this reason we will assume that there are at least two objects. We work in $\operatorname{ar}_{\Sigma}^{+}+\mathrm{E}_{2}^{\text {ob }}$. Let $x^{\star}$ and $y^{\star}$ be two distinct objects.

The class $Z$ is a sum of $X$ and $Y$ iff there are functions in $_{0}: X \rightarrow Z$ and $\operatorname{in}_{1}: X \rightarrow Z$ such that, for every $W$ and every $f: X \rightarrow W$ and every $g: Y \rightarrow W$ there is a unique $h$ such that $f=h \circ \mathrm{in}_{0}$ and $g=h \circ \mathrm{in}_{0}$. Clearly, $Z$ is uniquely determined up to isomorphism. We will write $X+Y$ for $Z$ and $[f, g]$ for $h$.

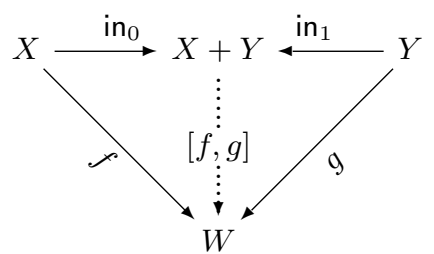

The sum need not always exist in our category. As usual, the sum is associative in the strong sense that, if $(X+Y)+Z$ exists, then $X+(Y+Z)$ exists and is isomorphic to $(X+Y)+Z$, and vice versa. The sum is also commutative in the strong sense. Finally, $X$ is a sum of $X$ and $\emptyset$, with in-arrows id $X$ and $\operatorname{emb}_{\emptyset, X}$.

We show that the sum, if it exists, is a disjoint union of isomorphic copies. First we show that the $\operatorname{in}_{i}$ are jointly injective. If $X$ is empty, the injectivity of $\operatorname{in}_{0}$ on $X$ is automatic. Suppose $x \in X$. take $W:=X, f:=\mathrm{id}_{X}, g: \operatorname{emb}_{\{x\}, X} \circ \mathrm{c}_{Y, x}$. We see that $[f, g] \circ \mathrm{in}_{0}=\mathrm{id}_{X}$. It follows that in $\mathrm{n}_{0}$ is injective. Similarly, we find that in $\mathrm{in}_{1}$ is injective. Finally, let $W:=\left\{x^{\star}, y^{\star}\right\}, f:=\operatorname{emb}_{\left\{x^{\star}\right\},\left\{x^{\star}, y^{\star}\right\}} \circ \mathrm{c}_{X, x^{\star}}, g:=\operatorname{emb}_{\left\{y^{\star}\right\},\left\{x^{\star}, y^{\star}\right\}} \circ \mathrm{c}_{Y, y^{\star}}$. We see that $[f, g]\left(\operatorname{in}_{0}(x)\right)=x^{\star}$ and $[f, g]\left(\operatorname{in}_{1}(y)\right)=y^{\star}$. So, $\operatorname{in}_{0}(x) \neq \operatorname{in}_{1}(y)$.

We show that $\mathrm{in}_{0}$ and $\mathrm{in}_{1}$ are jointly surjective. Suppose $z \in X+Y$ is not in the range of the $\mathrm{in}_{i}$. Consider our previous choice of $W, f$ and $g$. Suppose, e.g., $[f, g](z)=x^{\star}$. We easily see that $\left([f, g] \backslash\left\{\left(z, x^{\star}\right)\right\}\right) \cup\left\{\left(z, y^{\star}\right)\right\}$ exists (by BPC and adjunction) and also makes the sum diagram commute. This contradicts the uniqueness of $[f, g]$.

Let $X^{\prime}:=\operatorname{in}_{0}[X]$. This class exists by BPC as a subclass of $X+Y$. Let $\imath_{0}:=\operatorname{in}_{0} 1 X^{\prime}$. Since $\imath_{0}$ is injective and surjective, it is an isomorphism. Similarly, for $Y^{\prime}:=\operatorname{in}_{1}[Y]$. We may conclude that $X+Y$ is the disjoint union of $X^{\prime}$ and $Y^{\prime}$, which are isomorphic copies, respectively of $X$ and $Y$.

Conversely, it is easy to see that the union of two disjoint isomorphic copies of $X$ and $Y$, if such exist, is a sum. So, we have shown that sums are disjoint unions of isomorphic copies, where the sum exists iff there exist disjoint copies.

4.7. The Product. We work again in $\operatorname{ar}_{\Sigma}^{+}+\mathrm{E}_{2}^{\mathrm{ob}}$. The class $Z$ is a product of $X$ and $Y$ iff there are functions $\pi_{0}: Z \rightarrow X$ and $\pi_{1}: Z \rightarrow X$ such that, for every $W$ and every $f: W \rightarrow X$ and every $g: W \rightarrow Y$ there is a unique $h$ such that $f=\pi_{0} \circ h$ and $g=\pi_{1} \circ h$. Clearly, $Z$ is uniquely determined up to isomorphism. We will write $X \times Y$ for $Z$ and $(f, g)$ for $h$.

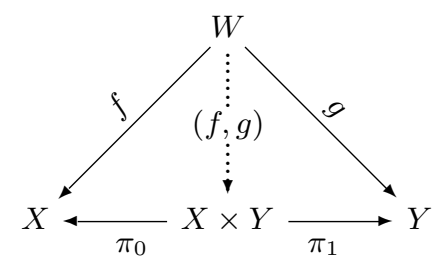

Note that we do not have the means available to develop the product as a set of pairs, since we cannot define an iterated pairing function on our domain. 
We can easily show that the product is associative and commutative, in the strong sense, and that $X \times \emptyset$ is $\emptyset$ and $X \times\{y\}$ is $X$. Also we have that, if $X \times Y$ and $X^{\prime} \times Y^{\prime}$ exist and $f: X \rightarrow X^{\prime}$ and $g: Y \rightarrow Y^{\prime}$, then there is a unique $f \times g: X \times Y \rightarrow Y \times Y^{\prime}$ with $f \circ \pi_{i}=\pi_{i}^{\prime} \circ(f \times g)$, for $i=0,1$. Moreover, when everything is defined $\times$ has the usual functorial properties.

We want to show the distributivity of + over $\times$. Suppose $X$ is not empty and $X \times Y$ exists. Say $x \in X$. The function $\jmath:=\left(\operatorname{emb}_{\{x\}, X} \circ \mathrm{c}_{Y, x}, \mathrm{id}_{Y}\right)$ is a coretraction: we have $\pi_{2} \circ \jmath=\mathrm{id}_{Y}$. It is immediate that $\jmath$ is injective. Let $Y^{\star}$ be $\jmath[Y]$. It follows that $\jmath^{\star}:=\jmath 1 Y^{\star}$ is a bijection from $Y$ to $Y^{\star}$ and, hence, that $Y$ and $Y^{\star}$ are isomorphic.

We assume that:

(1) $X$ is not empty.

(2) $X \times Y_{i}$ exists with witnessing projection functions $\pi_{j}^{i}$.

(3) $Z:=\left(X \times Y_{0}\right)+\left(X \times Y_{1}\right)$ exists.

We show that the class $\left(X \times Y_{0}\right)+\left(X \times Y_{1}\right)$ is a product of the form $X \times\left(Y_{0}+Y_{1}\right)$.

We first show that $Y_{0}+Y_{1}$ exists. Let $\jmath_{i}$ be the embedding of $Y_{i}$ in $X \times Y_{i}$ described above (for some fixed $x \in X$ ). We define:

- $Y_{0}+Y_{1}:=\operatorname{in}_{0} \jmath_{0}\left[Y_{0}\right] \cup \operatorname{in}_{1} \jmath_{1}\left(Y_{1}\right)$.

- $\mathrm{in}_{i}^{\prime}:=\mathrm{in}_{i} \circ \jmath_{i}$.

This is indeed the sum, since it is a union of disjoint isomorphic copies.

We show that $\left(X \times Y_{0}\right)+\left(X \times Y_{1}\right)$ is a product with witnessing projection functions $\pi_{0}^{*}:=\left[\pi_{0}^{0}, \pi_{0}^{1}\right]$ and $\pi_{1}^{*}:=\pi_{1}^{0}+\pi_{1}^{1}$. Here are the relevant diagrams.
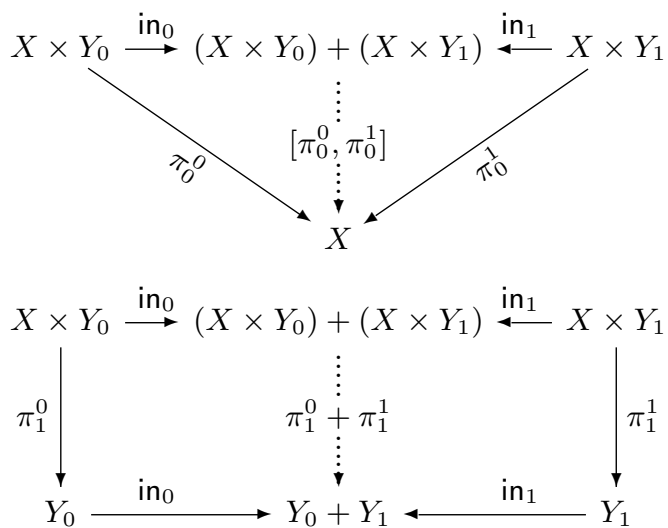

Suppose we have $f: W \rightarrow X$ and $g: W \rightarrow Y_{0}+Y_{1}$. Let $W_{i}:=g^{-1}\left[\operatorname{in}_{i}\left[Y_{i}\right]\right]$ and $g_{i}:=g 1 \operatorname{in}_{i}\left[Y_{i}\right]$. Clearly, the $W_{i}$ form a partition of $W$. We consider $W$ as the sum of the $W_{i}$ with the obvious embeddings as in-functions. Let $f_{i}:=f \uparrow W_{i}$. We find: $f=\left[f_{0}, f_{1}\right]$ and $g=g_{0}+g_{1}$. Putting everything together, we obtain the following diagram.

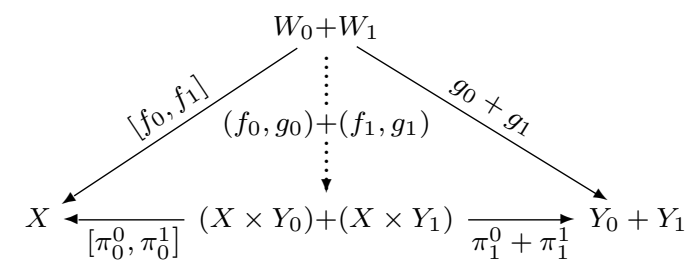

It is easily seen that the diagram commutes. To prove uniqueness, suppose $h$ also finishes the diagram. We have: $h:\left(W_{0}+W_{1}\right) \rightarrow\left(\left(X \times Y_{0}\right)+\left(X \times Y_{1}\right)\right.$. Let $h_{i}^{\prime}:=\left(h \uparrow W_{i}\right)$. Clearly the range of $h_{i}^{\prime}$ is $\operatorname{in}_{i}\left[X_{i} \times Y\right]$. Let $h_{i}:=h_{i}^{\prime} 1 \operatorname{in}_{i}\left[X_{i} \times Y\right]$. We find: $h=h_{0}+h_{1}$. We may conclude that $\pi_{0}^{i} \circ h_{i}=f_{i}$ and $\pi_{1}^{i} \circ h_{i}=g_{i}$. So $h_{i}=\left(f_{i}, g_{i}\right)$ and, thus, $h=\left(f_{0}, g_{0}\right)+\left(f_{1}, g_{1}\right)$. 
We turn to the other direction. Suppose that:

(1) $X$ is not empty.

(2) $Y_{0}+Y_{1}$ is defined.

(3) $X \times\left(Y_{0}+Y_{1}\right)$ is defined.

We show that $Z:=X \times\left(Y_{0}+Y_{1}\right)$ is a sum. Consider $Z_{i}:=\pi_{1}^{-1}\left[\operatorname{in}_{i}\left[Y_{i}\right]\right]$. We note that the $Z_{i}$ are disjoint and cover $X \times\left(Y_{0}+Y_{1}\right)$. Define $\pi_{j}^{i}:=\pi_{i} \uparrow Z_{i}$. We show that $Z_{i}$ is a product $X \times Y_{i}$. Suppose $f: W \rightarrow X$ and $g: W \rightarrow Y_{i}$. Then, we have $\left(\operatorname{in}_{i} \circ g\right): W \rightarrow\left(Y_{0}+Y_{1}\right)$. Let $h$ be the unique function given by the universal property of $X \times\left(Y_{0}+Y_{1}\right)$. It is easily seen that $h[W] \subseteq Z_{i}$. So $h 1 Z_{i}$ finishes the product diagram of $X \times Y_{i}$.

Let $h^{\prime}$ be another function that makes the diagram for $X \times Y_{i}$ and $f, g$ commute. It is easy to see that $\mathrm{emb}_{Z_{i}, Z} \circ h^{\prime}$ makes the diagram for $X \times\left(Y_{0}+Y_{1}\right)$ and $f, \mathrm{in}_{i} \circ g$ commute. So $h^{\prime}$ is uniquely determined.

4.8. Successor. We work in $\operatorname{ar}_{\Sigma}+\mathrm{E}_{2}^{\mathrm{ob}}$. A successor $\mathrm{S} X$ is simply a class $X+\{x\}$. We easily see that $\mathrm{S} X$ is defined iff there is an isomorphic copy $X^{\prime}$ of $X$ and an element $x^{\prime} \notin X^{\prime}$.

Suppose $\mathrm{S} X$ and $\mathrm{S} Y$ both exist and $\mathrm{S} X$ is isomorphic to $\mathrm{S} Y$. Say $\mathrm{S} X=X^{\prime} \cup\left\{x^{\prime}\right\}$, where $X^{\prime}$ is an isomorphic copy of $X$ and $x^{\prime} \notin X^{\prime}$. Say $\mathrm{S} Y=Y^{\prime} \cup\left\{y^{\prime}\right\}$, where $Y^{\prime}$ is an isomorphic copy of $Y$ and $y^{\prime} \notin Y^{\prime}$. It is easy to transform an isomorphism of $X^{\prime} \cup\left\{x^{\prime}\right\}$ and $Y^{\prime} \cup\left\{y^{\prime}\right\}$ into an isomorphism of $X^{\prime}$ and $Y^{\prime}$. Hence, $X$ and $Y$ are isomorphic. Ergo $\mathrm{S}$ is injective modulo isomorphism.

Finally, clearly, every $X$ is either the empty class or a successor.

4.9. Interpretation of Number Theory. We are now able to interpret $\mathrm{TN}_{0}$ in $\mathrm{ac}_{\Sigma}+\mathrm{E}_{2}^{\mathrm{ob}}$. First we interpret $\mathrm{ac}_{\Sigma}^{+}+\mathrm{E}_{2}^{\mathrm{ob}}$ in $\mathrm{ac} \mathrm{c}_{\Sigma}+\mathrm{E}_{2}^{\mathrm{ob}}$. Next, we interpret our numbers by the elements of $\mathcal{X}$ modulo isomorphism. We take $0:=\emptyset, \mathrm{S} X:=X+\{x\}$, and + and $\times$ are the + and $\times$ of the category. Note that we get more than $\mathrm{TN}_{0}$ : we have associativity and commutativity of addition and multiplication. We have the full distributivity of times over plus. (Since $\mathrm{TN}_{0}$ is finitely axiomatized, we get interpretability rather than local interpretability.)

Consider ac $\boxplus_{\mathfrak{o}}$ INF. This is, modulo some minor details, the theory $T_{0}$ of [Vau62]. It is immediate that this theory interprets $\mathrm{TN}_{\infty}$ and, hence $\mathrm{R} .{ }^{9}$ In the other direction, one can show that $\mathrm{R}$ interprets $\mathrm{ac} \boxplus_{\mathfrak{o}}$ INF. We will prove this in a subsequent paper.

Finally, consider ac extended with the no-universe axiom:

nu $\vdash \forall X \exists x x \notin X$.

In other words, we add the axiom: there is no universal class. We easily see that this theory implies that successor is total. Thus, it interprets $\mathrm{Q}_{\text {haj }}^{-}$, and, hence, Q. Conversely, it is well known that $Q$ interprets the theory AS of Subsection 5.3. Moreover, AS is easily seen to interpret ar + nu. Thus, $Q$ and ar + nu are mutually interpretable.

\section{Consequences}

In this section, we explore some consequences of our main result. Moreover, we discuss the relationship of our result to related results in the literature.

5.1. Progressive Linear Order. Let $U$ be any extension of $\operatorname{ar}_{\Sigma}$ that interprets the theory of a progressive linear preorder on the object domain, i.e. a linear preorder $L$ with the extra property $\forall x \in \delta_{L} \exists y x<_{L} y$. We now restrict the relations of our theory to the virtual class of relations $\mathcal{L}$, where $R$ is in $\mathcal{L}$ iff, for some $z \in \delta_{L}$, for all $x \in \delta_{L}$ and for all $y$, we have, if $R x y$ or $R y x$, then $x \leqslant L z$. I.o.w., the objects in the intersection of the field of $<_{L}$ and $\delta_{L}$, have an upper bound in $\delta_{L}$.

\footnotetext{
${ }^{9}$ This answers a question of Vaught, see [Vau62], p22.
} 
It is easy to see that $\mathcal{L}$ contains the empty relation and is closed under adjunction. Restricting our relations to $\mathcal{L}$ give us an o-direct interpretation of $\operatorname{ar}_{\Sigma}+$ nu. We may conclude that $U \triangleright \mathrm{Q}$.

5.2. A Total, Injective and non-Surjective Relation. The theory $\ln S$ of a total, injective and non-surjective binary relation is axiomatized by the follwoig axioms.

ins1. $\vdash \exists y \forall x \neg x \sigma y$

ins2. $\vdash \forall x \exists y x \sigma y$,

ins3. $\vdash \forall x, y, z((x \sigma z \wedge y \sigma z) \rightarrow x=y$.

There is another way of looking at the theory $\ln S$. We introduce Vaught's Set Theory VS. (This theory was introduced in [Vau67].) It's axioms are:

vs $_{n} . \vdash \forall x_{0}, \ldots, x_{n-1} \exists y \forall z\left(z \in y \leftrightarrow \bigvee_{i<n} z=x_{i}\right)$.

For the case $n=0$, $\mathbf{v s}_{0}$ gives us the existence of empty sets. The theory $\mathrm{VS}_{n}$ is axiomatized by the axioms $\mathbf{v s}_{0}$ and $\mathbf{v s}_{n}$. The theory VS is axiomatized by all axioms $\mathbf{v s}_{n}$. The theory VS interprets R. Since, R is locally finitely satisfiable, i.e., every finite subtheory has a finite model, it is clear that $\mathrm{R}$ does not even interpret $\mathrm{VS}_{1}$.

We claim that $\mathrm{VS}_{2}$ is mutually o-directly interpretable with $\ln S$. To interpret $\mathrm{VS}_{2}$ in InS, it is sufficient to read translate $x \in y$ by $y \sigma x$. In the other direction, we translate $\sigma$ by:

- $y \sigma x: \leftrightarrow y \in x \wedge \forall z \in_{0} x z=y$.

We start with ar $\boxplus_{\mathfrak{o}} \operatorname{InS}$. We have shown how to o-directly interpret $\mathrm{ar}^{+}$in ar. In this case we need only add: subtraction of singleton relations. We work in $\operatorname{ar} \boxplus_{\mathfrak{o}} \ln S$ plus subtraction of singleton relations.

Define the virtual class $\mathcal{Z}$ as the class of all classes $Z$ such that:

i. $Z$ is Dedekind finite, i.o.w, if $Z \equiv Y$ and $Y \subseteq Z$, then $Y=Z$. Here $\equiv$ means that there is a witnessing bijection.

ii. There is an $Y$ and an $f: Z \equiv Y$, such that for all $z$ in $Z$, we have $z \sigma f z$.

Clearly, $\mathcal{Z}$ contains the empty class. We show that it is closed under adjunction.

We verify the preservation of (i). Suppose $X$ is in $\mathcal{Z}$. Consider $X \cup\{x\}$. If $x \in X$, we are done. So, suppose $x \notin X$. Suppose $g:(X \cup\{x\}) \equiv Y$ and $Y \subseteq(X \cup\{x\})$. If $x \in Y$, we define $Y^{\prime}:=Y$ and $g^{\prime}:=g$. If $x \notin Y$, we take $Y^{\prime}:=(Y \backslash\{g x\}) \cup\{x\}$ and $g^{\prime}:=(g \backslash\{(x, g x)\}) \cup\{(x, x)\}$. We find $g^{\prime}: X \equiv Y^{\prime}, Y^{\prime} \subseteq(X \cup\{x\})$, and $x \in Y^{\prime}$. It follows that:

$$
\left(g^{\prime} \backslash\left\{\left(x, g^{\prime} x\right)\right\}\right):(X \backslash\{x\}) \equiv\left(Y \backslash\left\{g^{\prime} x\right\}\right)
$$

So, $X=Y^{\prime} \backslash\{x\}$ and, hence, $X \cup\{x\}=Y^{\prime}$. If $Y \neq Y^{\prime}$, we have: $g x \notin Y^{\prime}$. But, $g x \in Y \subseteq(X \cup\{x\})=Y^{\prime}$. A contradiction. So $X \cup\{x\}=Y^{\prime}=Y$.

The preservation of (ii) under adjunction is easy.

We claim that the universe, say $V$, is not in $\mathcal{Z}$. Suppose $\mathcal{Z}$ did contain $V$. We would have $f: V \equiv Y$, where $f$ is contained in the virtual relation $\sigma$. Since $\sigma$ is not surjective, we find $Y \neq V$. On the other hand $Y \subseteq V$, contradicting the Dedekind finiteness of $V$.

We restrict our relations to the union of $\mathcal{Z}$ and the non-diagonal relations. This gives us a direct interpretation witnessing: $\left(\operatorname{ar} \boxplus_{\mathfrak{o}} \ln S\right) \triangleright_{\mathfrak{o} \text {-dir }}(\mathrm{ar}+\mathrm{nu})$. It immediately follows that $\left(\operatorname{ar} \boxplus_{\mathfrak{o}} \mathrm{VS}_{1}\right) \triangleright_{\mathfrak{o} \text {-dir }}(\operatorname{ar}+\mathrm{nu})$. Thus we find that $\left(\operatorname{ar} \boxplus_{\mathfrak{o}} \ln S\right) \triangleright_{\mathfrak{o} \text {-dir }} \mathrm{Q}$ and $\left(\operatorname{ar} \boxplus_{\mathfrak{o}} \vee S_{1}\right) \triangleright_{\mathfrak{o} \text {-dir }} \mathrm{Q}$.

Remark 5.1. There is an alternative strategy for obtaining this result. Note that, as soon as we have developed our category in $\mathrm{ar}^{+}$, it is sufficient for the totality of successor that every class is isomorphic to a non-universe. To get this property, it is sufficient to ensure that, for every $Z$, there are $Y$ and $f$ such that $f: Z \equiv Y$ and, for all $z$ in $Z, z \sigma f z$. If we pursue this strategy, we must make sure that the result of restricting the classes is closed under isomorphic copies. 
Remark 5.2. We can improve our result by only demanding that we have the InS-axioms on a (sub)domain of objects $\delta$ modulo an equivalence relation $E$.

Example 5.3. Let SUCC be the theory with the following axioms:

succ $1 \vdash \mathrm{S} x \neq 0$.

succ2 $\vdash \mathrm{S} x=\mathrm{S} y \rightarrow x=y$.

John Burgess proves in [Bur05] that $\left(\operatorname{ar} \boxplus_{\mathfrak{o}}\right.$ SUCC) $\triangleright$ Q. This result follows immediately from the result of this section, since $\mathbf{S} x=y$ is a total, injective and non-surjective relation.

5.3. Sets versus Pairs \& Classes. In this subsection, we study adjunctive set theory (sometimes also called: baby set theory) and the adjunctive theory of classes over a basic theory of pairs. First we introduce adjunctive set theory AS. The language of the onesorted theory AS has, apart from identity, just one binary predicate $\in$. The theory is given by the following axioms.

AS1. $\vdash \exists x \forall y y \notin x$,

AS2. $\vdash \forall x, y \exists z \forall u(u \in z \leftrightarrow(u \in x \vee u=y))$.

As discussed in the introduction, AS is the fundamental theory used to define the notion of sequentiality. The adjunctive theory of classes ac is a close analogue of ar. It is a two sorted theory of objects and classes. We have, apart from identity the binary predicate app of type co. We write $x \in X$ for app $(X, x)$. Here are the axioms of ac.

ac1. $\vdash \exists X \forall y y \notin X$,

ac2. $\vdash \forall X, y \exists Z \forall u(u \in Z \leftrightarrow(u \in X \vee u=y))$,

ac3. $\vdash \forall X, Y(\forall z(z \in X \leftrightarrow z \in Y) \rightarrow X=Y)$.

The theory of non-surjective unordered pairing and a theory of non-surjective ordered pairing is the theory $\mathrm{VS}_{2} \cdot{ }^{10}$ This theory is mutually o-directly interpretable with a theory PAIR of non-surjective ordered pairing which is defined as follows.

pair1. $\vdash \exists z \forall x, y \neg$ pair $(x, y, z)$,

pair2. $\vdash \forall x, y \exists z$ pair $(x, y, z)$,

pair3. $\vdash \forall x, x^{\prime}, y, y^{\prime}, z\left(\left(\operatorname{pair}(x, y, z) \wedge \operatorname{pair}\left(x^{\prime}, y^{\prime}, z\right)\right) \rightarrow\left(x=x^{\prime} \wedge y=y^{\prime}\right)\right)$.

We can directly interpret $\mathrm{VS}_{2}$ in PAIR, by translating $x \in y$ to the formula:

$$
\exists u(\operatorname{pair}(x, u, y) \vee \operatorname{pair}(u, x, y)) .
$$

We can directly interpret PAIR in $\mathrm{VS}_{2}$ via Wiener-Kuratowski pairing. We translate pair $(x, y, z)$ into:

$$
\begin{aligned}
& \exists u, v(\forall w(w \in z \leftrightarrow(w=u \vee w=v)) \wedge \\
& \left.\forall w^{\prime}\left(w^{\prime} \in u \leftrightarrow w^{\prime}=x\right) \wedge \forall w^{\prime \prime}\left(w^{\prime \prime} \in v \leftrightarrow\left(w^{\prime \prime}=x \vee w^{\prime \prime}=y\right)\right)\right) .
\end{aligned}
$$

We have:

Theorem 5.4. $\left(\mathrm{ac} \boxplus_{\mathfrak{o}} \mathrm{PAIR}\right) \equiv_{\mathfrak{o}-\text { dir }}$ AS.

Proof. The theory AS o-directly interprets ( $\mathrm{ac} \boxplus_{\mathfrak{o}}$ PAIR) using diagonal relations and Wiener-Kuratowski pairing.

We prove that $\left(\mathrm{ac} \boxplus_{\mathfrak{o}} \mathrm{PAIR}\right) \triangleright_{\mathfrak{o} \text {-dir }} \mathrm{AS}$. To give the heuristic, let's ignore for a moment the fact that pairing is not necessarily functional. The basic idea is to code e.g. the set consisting of $a, b, c$ as $\langle\langle\langle 0, a\rangle, b\rangle, c\rangle$, where 0 is a non-pair. Now forget about functionality again. We define:

- $\mathrm{dc}(Y): \leftrightarrow \forall u, v, p((\operatorname{pair}(u, v, p) \wedge p \in Y) \rightarrow u \in Y)$,

(We will also write $Y$ :dc for $\mathrm{dc}(Y)$.)

- $x \in y: \leftrightarrow \forall Y: \mathrm{dc}(y \in Y \rightarrow \exists w, q(\operatorname{pair}(w, x, q) \wedge q \in Y))$.

\footnotetext{
${ }^{10}$ John Burgess in [Bur05] calls the result of adding extensionality to this theory: UST.
} 
Consider any non-pair $z$. We clearly have $\{z\}$ :dc. If we would have $x \in z$, then, for some pair $q$, we would have that $q$ is in the class $\{z\}$, quod non. So $z$ is an empty set.

Consider any $x$ and $y$. Pick any $p$ with pair $(y, x, p)$. We show that:

$$
\forall u(u \in p \leftrightarrow(u \in y \vee u=x)) .
$$

We first treat the right-to-left direction. Suppose $u \in y, \mathrm{dc}(\mathrm{Y})$ and $p \in Y$. We find $y \in Y$, and, hence, for some $w$ and $q$, $\operatorname{pair}(w, u, q)$ and $q \in Y$. So, $u \in p$. Moreover, it is immediate that $x \in p$.

Conversely, suppose $u \in p, \operatorname{dc}(Y)$ and $y \in Y$. We have $\operatorname{dc}(Y \cup\{p\})$ and $p \in Y \cup\{p\}$. It follows that, for some $w$ and $q$, we have pair $(w, u, q)$ and $q \in Y \cup\{p\}$. If $q=p$, then $u=x$. If $q \neq p$, then $q \in Y$ and, thus, $u \in y$.

The above theorem illustrates that the 'direct' sum $\boxplus_{\mathfrak{o}}$ makes the summands interact in non-trivial ways. In this, it contrasts with the ordinary disjoint sum $\oplus$. E.g., sequential theories like AS are connected or join-irreducible in the degrees of interpretability. See [Pud83] and [Ste89].

Here is our new proof of the (Tarski+Szmielew) - (Collins+Halpern) - (Montagna+Mancini) - (Mycielski+Pudlák+Stern) Theorem.

Theorem 5.5. Each of $\mathrm{ac} \boxplus_{\mathfrak{o}} \mathrm{VS}_{2}$, ac $\boxplus_{\mathfrak{o}} \mathrm{PAIR}$, and AS interprets $\mathrm{Q}$.

Proof. Since, by our earlier results, the theories ac $\boxplus_{\mathfrak{o}} \mathrm{VS}_{2}, \mathrm{ac}_{\mathfrak{o}} \mathrm{PAIR}$, and AS are mutually $\mathfrak{o}$-directly interpretable, it is sufficient to show that one of them interprets $Q$. We evidently have $\left(\mathrm{ac} \boxplus_{\mathfrak{o}} \mathrm{VS}_{2}\right) \triangleright_{\mathfrak{o} \text {-dir }}\left(\operatorname{ar} \boxplus_{\mathfrak{o}} \mathrm{VS}_{1}\right)$, since we can interpret the relations as classes of pairs. So, by the result of Subsection 5.2, we are done.

\section{Separations}

We separate some of the salient systems of this paper in the preorder of $\mathfrak{o}$-direct interpretability.

Theorem 6.1. We have $\mathrm{EQ} \oiint_{\mathfrak{o} \text {-dir }}$ ac $\oiint_{\mathfrak{o} \text {-dir }}$ ar

Proof. The fact that EQ $\triangleleft_{\mathfrak{o} \text {-dir }}$ ac $\triangleleft_{\mathfrak{o} \text {-dir }}$ ar is easy.

Suppose $E Q \triangleright_{\mathfrak{o}-\text { dir }}$ ac. Then:

$$
\begin{array}{rll}
\text { PAIR } & = & \text { EQ } \boxplus_{\mathfrak{o}} \text { PAIR } \\
\triangleright_{\mathfrak{o} \text {-dir }} & \text { ac } \boxplus_{\mathfrak{o}} \text { PAIR } \\
\equiv_{\mathfrak{o} \text {-dir }} & \text { AS }
\end{array}
$$

However, PAIR has a decidable extension (see e.g., [Ten] or [CR01]) and AS is essentially undecidable. Quod impossibile.

Here is another, relatively theory-free, proof of the same fact. Suppose we have an o-direct interpretation $K$ of ac in EQ. Let's allow more-dimensional and piece-wise interpretations, to create the classes. (This rules out the one-element model as a trivial counterexample.) It is easy to see that there are $k$ and $m$ such that for every EQ-model $\mathcal{M}$ with $n$ elements, the internal model $K(\mathcal{M})$ has at most $n^{k}+m$ elements. But an ac-model with $n$-objects has $2^{n}$ classes. A contradiction.

Suppose ac $\triangleright_{\mathfrak{o}-\text { dir }}$ ar. Then:

$$
\begin{array}{cll}
\text { ac } \boxplus S U C C & \triangleright_{\mathfrak{o} \text {-dir }} & \text { ar } \boxplus \text { SUCC } \\
\triangleright & Q
\end{array}
$$

It follows that ac $\boxplus_{\mathfrak{o}}$ SUCC is essentially undecidable. On the other hand, ac $\boxplus_{\mathfrak{o}}$ SUCC is contained in the (true) monadic second order theory of one successor. This theory is decidable. A contradiction. Alternatively, ac $\boxplus_{\mathfrak{o}}$ SUCC is contained in the weak (true) 
monadic second order theory of one successor. Here the second order variables range over finite sets. This theory is again decidable. Again we have our contradiction. (See [BE59], [Büc60], [Elg61], [ER66], for the basics on weak and strong successor theories.)

Here is an alternative, theory-free, proof. Suppose we have an o-direct interpretation $K$ of ar in ac. There is a $k$ such that, for any model $\mathcal{M}$ of ac with $N$ elements, the number of elements of $K(\mathcal{M})$ is of order $N^{k}$. Moreover, if $\mathcal{M}$ has $n$ objects, then the number of elements (objects and classes) of $\mathcal{M}$ is of order $2^{n}$. So, $K(\mathcal{M})$ has about $2^{k n}$ elements. On the other hand the number of elements of a model of ar with $n$ objects is of order $2^{n^{2}}$. A contradiction.

Theorem 6.2. We have:

$$
\begin{aligned}
& (\mathrm{ar}+\mathrm{nu}) \supsetneqq_{\mathrm{o}-\mathrm{dir}} \quad\left(\mathrm{ar} \boxplus_{\mathfrak{o}} \mathrm{VS}_{1}\right) \\
& \equiv_{\mathfrak{o}-\text { dir }} \quad\left(\operatorname{ar} \boxplus_{\mathfrak{o}} \ln S\right) \\
& ¥_{\mathrm{o}-\mathrm{dir}} \quad\left(\mathrm{ac} \boxplus_{\mathrm{o}} \mathrm{VS}_{2}\right) \\
& \equiv_{\mathfrak{o}-\text { dir }} \quad\left(\text { ac } \boxplus_{\mathfrak{o}} \text { PAIR }\right) \\
& \equiv_{\mathfrak{o}-\operatorname{dir}} \text { AS. }
\end{aligned}
$$

Proof. We show that ar + nu does not o-directly interpret ar $\boxplus_{\mathfrak{o}} \mathrm{VS}_{1}$, even when we allow ourselves parameters. Consider the model $\mathcal{M}$ of ar + nu, where we have an infinite domain of urelements and as classes the finite sets over that domain. Suppose we have finitely many parameters $\vec{p}$. Without loss of generality we may assume that these are urelements. Suppose we had an o-direct interpretation $K$ of $\operatorname{ar} \boxplus_{\mathfrak{o}} \mathrm{VS}_{2}$. Let $\mathcal{V}_{0}$ be the virtual class of urelements that are not set-singletons, $\mathcal{V}_{1}$ the virtual class of urelements that are setsingletons of the elements of $\mathcal{V}_{0}$, etc. All these classes are disjoint and non-empty. Consider any $\mathcal{V}_{k}$ without elements from $\vec{p}$. Since all permutations of the urelements that fix $\vec{p}$ induce automorphisms of our model, we find that $\mathcal{V}_{k}$ must include all urelements except possibly some parameters. But this must hold of co-finitely $\mathcal{V}_{k}$. A contradiction.

We show that $\operatorname{ar} \boxplus_{\mathfrak{o}} \mathrm{VS}_{1}$ does not $\mathfrak{o}$-directly interpret ac $\boxplus_{\mathfrak{o}} \mathrm{PAIR}$. Consider the following model $\mathcal{N}$ of $\operatorname{ar} \boxplus_{\mathfrak{o}} \mathrm{VS}_{1}$. The elements of the domain sequences of $0,1,2$ including the empty sequence. We define $s \in t$ iff $t=s j$, for $j=0,1,2$. We add all finite relations over our domain. We show that we cannot define pairing over the object domain in $\mathcal{N}$, not even with parameters. Let the parameters be $\vec{u}$. Suppose we could define pairing. Clearly, we can find $s 0, s 1$ and $t$ such that pair $(s 0, s 1, t)$ and the si and $t$ are all longer than the $\vec{u}$.

Let $\alpha$ be a permutation of $\{0,1,2\}$. We define $\sigma_{w, \alpha}(v):=w \alpha(i) v_{1}$, if $v=w i v_{1}$, and $\sigma_{w, \alpha}(v):=v$, otherwise. We easily see that $\sigma_{w, \alpha}$ lifts to an automorphism of $\mathcal{N}$, which, par abus de langage, we will again call ' $\sigma_{w, \alpha}$ '.

If $t$ is not a weak end-extension of one of $s 0, s 1$, we find that $\sigma_{s,(01)}$ interchanges the si and leaves $t$ in place. If $t$ is a weak extension of, say, $s 0$, then $\sigma_{s,(12)}$ leaves $t$ in place but sends $s 1$ to $s 2$. However, the pairing axiom tells us that any automorphism that fixes $t$ also should fix $s 0$ and $s 1$. A contradiction.

Note that it follows from the above considerations that $\mathrm{VS}_{1} \triangleright_{\mathrm{o} \text {-dir }} \mathrm{VS}_{2}$. On the other hand, it is easy to see that $\mathrm{VS}_{2} \triangleright_{\mathfrak{o} \text {-dir }} \mathrm{VS}_{n}$, for all $n$, and so $\mathrm{VS}_{2} \triangleright_{\mathfrak{o} \text {-dir,loc }} \mathrm{VS}$. We do not have $\mathrm{VS}_{2} \triangleright \mathrm{VS}$, since $\mathrm{VS}_{2}$ has decidable extensions and VS is essentially undecidable.

\section{REFERENCES}

[BE59] J.R. Büchi and C.C. Elgot. Decision problems of of weak second order arithmetics and finite automata, part I (abstact). American Mathematical Society Notices, 5:834, 1959.

[Büc60] J.R. Büchi. Weak second order arithmetic and finite automata. Zeitschrift für Mathematische Logik und Grundlagen der Mathematik, 6:66-92, 1960.

[Bur05] John Burgess. Fixing Frege. Princeton Monographs in Philosophy. Princeton University Press, Princeton, 2005. 
[CH70] G.E. Collins and J.D. Halpern. On the interpretability of Arithmetic in Set Theory. The Notre Dame Journal of Formal Logic, 11:477-483, 1970.

[CR01] P. Cégielski and D. Richard. Decidability of the natural integers equipped with the Cantor pairing function and successor. Theoretical Computer Science, 257, 1-2:51-77, 2001.

[Elg61] C.C. Elgot. Decision problems of finite automata design and related arithmetics. Transactions of the American Mathematical Society, 98:21-51, 1961.

[ER66] C.C. Elgot and M.O. Rabin. Decidability and Undecidability of Extensions of Second (First) Order Theory of (Generalized) Successor. Journal of Symbolic Logic, 31:169-181, 1966.

[HP91] P. Hájek and P. Pudlák. Metamathematics of First-Order Arithmetic. Perspectives in Mathematical Logic. Springer, Berlin, 1991.

[JS83] J. P. Jones and J.C. Shepherdson. Variants of Robinson's essentially undecidable theory r. Archiv für Mathematische Logik und Grundlagenforschung, 23:61-64, 1983.

[MM94] F. Montagna and A. Mancini. A minimal predicative set theory. The Notre Dame Journal of Formal Logic, 35:186-203, 1994.

[MPS90] J. Mycielski, P. Pudlák, and A.S. Stern. A lattice of chapters of mathematics (interpretations between theorems), volume 426 of Memoirs of the American Mathematical Society. AMS, Providence, Rhode Island, 1990.

[Nel86] E. Nelson. Predicative arithmetic. Princeton University Press, Princeton, 1986.

[Pud83] P. Pudlák. Some prime elements in the lattice of interpretability types. Transactions of the American Mathematical Society, 280:255-275, 1983.

[Solle] R. M. Solovay. Interpretability in set theories. Unpublished letter to P. Hájek, 1976, Aug. 17. $\left\langle\right.$ http://www.cs.cas.cz/ ${ }^{\sim}$ hajek/RSolovayZFGB.pdf $\rangle$.

[ST50] W. Szmielew and A. Tarski. Mutual Interpretability of some essentially undecidable theories. In Proceedings of the International Congress of Mathematicians (Cambridge, Massachusetts, 1950), volume 1, page 734. American Mathematical Society, Providence, 1950 .

[Ste89] A.S. Stern. Sequential theories and infinite distributivity in the lattice of chapters. Journal of Philosophical Logic, 54:190-206, 1989.

[Šve07] V. Švejdar. An interpretation of Robinson's Arithmetic in Grzegorczyk's weaker variant. Fundamenta Informaticae, 81:347-354, 2007.

[Ten] R.L. Tenney. Decidable pairing functions. Unpublished.

[TMR53] A. Tarski, A. Mostowski, and R.M. Robinson. Undecidable theories. North-Holland, Amsterdam, 1953.

[Vau62] R.L. Vaught. On a theorem of cobham concerning undecidable theories. In E. Nagel, P. Suppes, and A. Tarski, editors, Logic, Methodology and Philosophy of Science. Proceedings of the 1960 International Congress, pages 14-25. Stanford University Press, Stanford, 1962.

[Vau67] R.A. Vaught. Axiomatizability by a schema. The Journal of Symbolic Logic, 32(4):473479, 1967.

[Vis08] A. Visser. Pairs, sets and sequences in first order theories. Archive for Mathematical Logic, 47(4):299-326, 2008.

Department of Philosophy, Utrecht University, Heidelberglaan 8, 3584 CS Utrecht, The NetherLands

E-mail address: Albert.Visser@phil.uu.nl 